\title{
Design of an underwater sensor network perpetually powered from AUVs
}

\author{
Luís M. Pessoa \\ INESC TEC \\ Porto, Portugal \\ luis.m.pessoa@inesctec.pt
}

\author{
Cândido Duarte \\ INESC TEC and FEUP \\ Porto, Portugal \\ candidoduarte@fe.up.pt
}

\author{
Henrique M. Salgado \\ INESC TEC and FEUP \\ Porto, Portugal \\ hsalgado@fe.up.pt
}

\author{
Vasco Correia \\ INESC TEC \\ Porto, Portugal \\ vasco.m.correia@inesctec.pt
}

\author{
Bruno Ferreira \\ INESC TEC and FEUP \\ Porto, Portugal \\ bmferreira@fe.up.pt
}

\author{
Nuno A. Cruz \\ INESC TEC and FEUP \\ Porto, Portugal \\ nacruz@fe.up.pt
}

\author{
Anibal Matos \\ INESC TEC and FEUP \\ Porto, Portugal \\ anibal@fe.up.pt
}

\begin{abstract}
In this paper we evaluate the long-term deployment feasibility of a large-scale network of abandoned underwater sensors, where power is provided by autonomous underwater vehicles (AUVs) in periodic visits.
\end{abstract}

Keywords-AUV, underwater sensor network, long-term deployment

\section{INTRODUCTION}

The Atlantic Ocean's characteristics and dimensions establish a set of scientific challenges to marine technologies, including underwater tools and sensors in the field of deep sea exploration and environmental monitoring due to the demanding operating conditions. Monitoring such a vast area represents a heavy financial burden resulting from the allocation of costly means (e.g., research vessel time). The employment of sensors within underwater environments is nowadays a standard practice in several fields of activity, aiming at collecting data on water or seabed parameters, as well as for the monitoring of permanent subsea infrastructures. These sensors may be simply abandoned on the seabed or located in fixed or mobile structures, the latter most often constituted by remotely operated vehicles (ROVs). The current solution for collecting data underwater involves the operation of ROVs, which is very expensive since a support vessel is required, and therefore can only be considered for small-scale operations. Therefore, there is a need for new scalable technological solutions to enable the collection of underwater sensor data, otherwise it will be economically impractical to monitor large remote oceanic areas. Autonomous Underwater Vehicles (AUVs) are an emerging and proven scalable solution, suitable for autonomous operation, constituting an affordable option to collect underwater sensor data. Figure 1 shows the envisioned scenario of an underwater long-term deployment consisting of a given set of abandoned underwater sensors. In this scenario, an AUV can approach several sensors to collect their data, and recharge their batteries, providing them with enough energy until the next visit. The AUV can recharge its own battery in an underwater docking station.

In this paper we evaluate the feasibility of long-term deployment for an abandoned network of underwater sensors, periodically visited by an AUV to provide energy and gather measurement data. We perform a scalability analysis in order to understand what size of network could be supported by a single AUV, both in terms of total number of sensors as well as separation distance between sensors.

The paper is organized as follows: section 2 describes the characteristics of the considered underwater sensor network, section 3 describes the mechanisms for AUV - sensor approach and the parameters considered in the performed analysis, section 4 describes results of the performed analysis, and section 5 provides the conclusion.

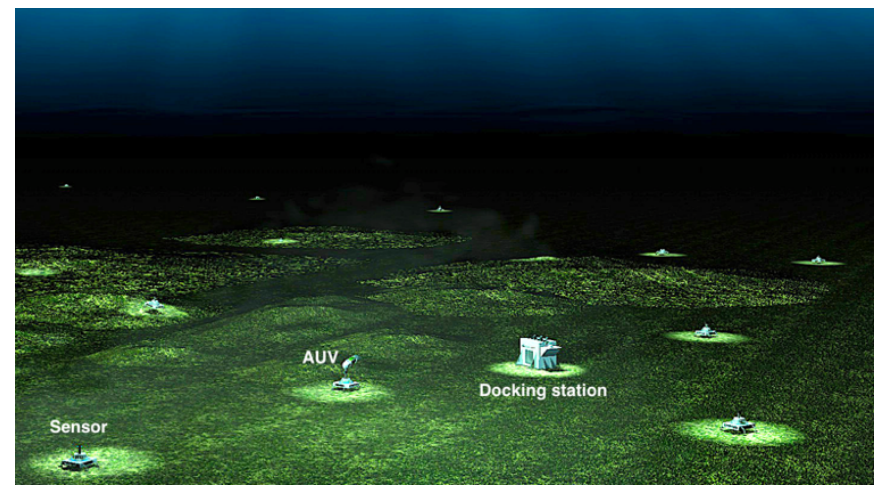

Figure 1 - Overview of the envisioned underwater long-term deployment scenario. 


\section{UNDERWATER SENSOR NETWORK}

The underwater sensor network is considered to have an homogenous geographical distribution across the seabed in the form of a linear string or alternatively with multiple rows in a matrix form as shown in Figure 2, where the distance between sensors $\left(d_{s}\right)$ is defined as a linear variable ranging from 100 metres to 2000 metres.

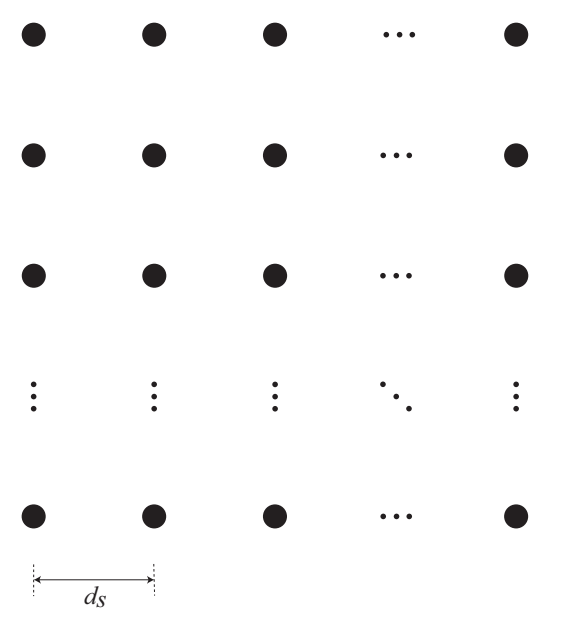

Figure 2 - Geographical distribution of the considered underwater sensor network.

We assume that each sensor is equipped with (1) a wireless power transfer (WPT) receiver [1], which allows it to receive power wirelessly from an AUV, and (2) an optical wireless communications (OWC)e transceiver [2], which allows for collected sensor data to be transferred quickly to an AUV at a data-rate of $10 \mathrm{Mbit} / \mathrm{s}$. We consider an average sensor power consumption ranging from $100 \mathrm{~mW}$ to $1 \mathrm{~W}$, already taking into account the typical duty cycled operation of underwater sensors, as well as an average total data size of $100 \mathrm{Mbit}$ to be collected in each visit. However, our analysis could be extended to other ranges of sensor power consumption.

The following operation cycle is assumed: The AUV departs from the docking station where it stayed until its battery became fully charged ( 2 hours of battery charging time are considered). Then the AUV travels to the nearest sensor, approaches it, and provides energy to the sensor via the wireless power transfer mechanism. During this time, the AUV establishes communication with the sensor via optical wireless communications to collect the stored sensor data. After the sensor receives enough energy (sufficient for ensuring its operation until the next visit), the AUV moves to the next sensor. We consider that the AUV returns to the docking station after visiting all the sensors. We also assume that the AUV battery capacity should be equal to the total energy spent by the AUV in one period of operation, where the all the sensors are visited.

\section{AUV - SENSOR APPROACH MECHANISM AND PARAMETERS}

For the present analysis, we consider the employment of the MARES AUV, a highly flexible small-scale AUV with hovering capability [3]. Table I describes the different parameters considered for the analysis, related to both the considered AUV and sensors.

TABLE I. SUMMARY OF PARAMETERS AND VALUES CONSIDERED IN THE PERFORMED ANALYSIS

\begin{tabular}{|l|l|}
\hline Parameter & Considered Value \\
\hline AUV battery capacity & $600 \mathrm{Wh}$ \\
\hline $\begin{array}{l}\text { AUV energy consumption at cruise speed }(1 \\
\text { m/s) }\end{array}$ & $50 \mathrm{~W}$ \\
\hline AUV energy consumption while hovering & $30 \mathrm{~W}$ \\
\hline AUV battery recharge duration & 2 hours \\
\hline WPT transmission power & $200 \mathrm{~W}$ \\
\hline WPT efficiency & $20 \%$ \\
\hline $\begin{array}{l}\text { OWC instantaneous power consumption for } \\
\text { data retrieval }\end{array}$ & $10 \mathrm{~W}$ \\
\hline OWC data-rate & $10 \mathrm{Mbit} / \mathrm{s}$ \\
\hline Sensor data collection size per visit & $100 \mathrm{Mbit}$ \\
\hline Sensor average power consumption & $100 \mathrm{~mW}-1 \mathrm{~W}$ \\
\hline
\end{tabular}

Figure 3 shows an AUV in the proximity of a sensor during the process of energy transfer and communications with the sensor.

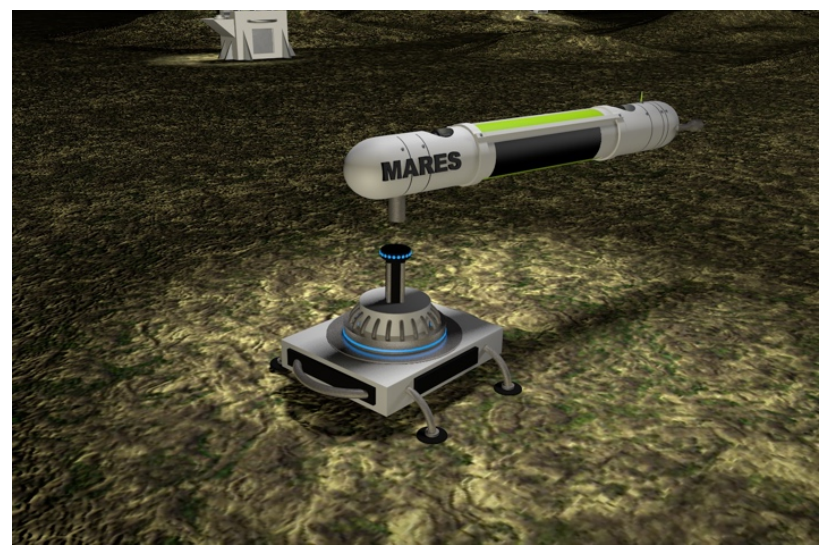

Figure 3 - Overview of the AUV while approaching an underwater sensor.

The following process of approach to each sensor is considered:

1. The AUV approaches the area where the sensor is located using an acoustic beacon.

2. After approaching the sensor within $\sim 3$ metres of proximity, the AUV sends an acoustic command requesting the activation of the light emitting diodes (LEDs) from the OWC system at the sensor.

3. The sensor receives the acoustic command and activates LEDs.

4. AUV performs a precision navigation guided by the LEDs located at the sensor which allows it to achieve a final position with an accuracy of $50 \mathrm{~cm}$ in the $x y$ plane and $10 \mathrm{~cm}$ accuracy in the $z$ axis [4].

5. After reaching the final position, the AUV activates the WPT system in order to transfer energy to the sensor battery. Given the achieved $z$ axis accuracy of $10 \mathrm{~cm}$, a safe separation of $30 \mathrm{~cm}$ is assumed between the AUV and sensor, which leads to the WPT efficiency of $20 \%$ considered in the analysis.

6. The sensor activates the OWC system and starts sending data to the AUV. The AUV receives the data 
and stores it in memory. A total sensor data collection size per visit of $100 \mathrm{Mbit}$ is considered.

7. AUV detects that enough energy has been transferred to the sensor, and considers that sensor energization is complete.

8. The AUV navigates away from the sensor and proceeds to the next sensor to be visited.

\section{RESULTS}

We start the analysis by evaluating the sensor required energy in each visit. The energy that the sensor requires depends on the the sensor power consumption as well as on the AUV re-visit time (the time between two consecutive visits). The re-visit period corresponds to the total time the AUV takes to visit all sensors plus the AUV battery recharge (considered 2 hours). Since the total time the AUV takes to visit all sensors also depends on the sensor required energy per visit, we considered an initial estimate of 10 hours of re-visit period, and followed a simple iterative adjustment to converge to the final values (4 iterations were considered). Figure 4 shows the final converged values of sensor required energy as a function of the distance between sensors, for different average sensor power consumption values of $100 \mathrm{~mW}, 500 \mathrm{~mW}$ and $1 \mathrm{~W}$.

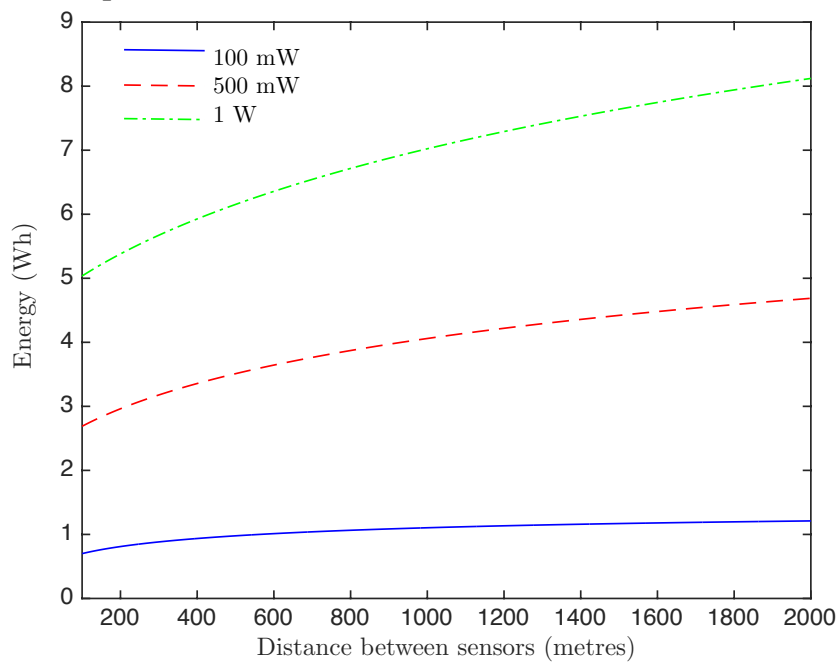

Figure 4 - Required energy per sensor as a function of the distance between sensors, for different average sensor power consumption values of $100 \mathrm{~mW}$, $500 \mathrm{~mW}$ and $1 \mathrm{~W}$.

It can be observed that the sensor required energy does not increase linearly with the sensor consumption. In order to understand the reason for this, we evaluated the total number of sensors and total time required for the AUV to approach all sensors, as a function of the distance between sensors, for different average sensor power consumption values of $100 \mathrm{~mW}$, $500 \mathrm{~mW}$ and $1 \mathrm{~W}$, as shown in Figure 5. It can be observed that the total time required for the AUV to approach all sensors reduces significantly with the increase of the sensor power consumption, since the total number of sensors that can be visited is also reduced due to the increased energy expenditure at each sensor, both in terms of energy delivered to the sensor as well as in terms of energy spent by the increased time the AUV needs to be hovering near the sensor.

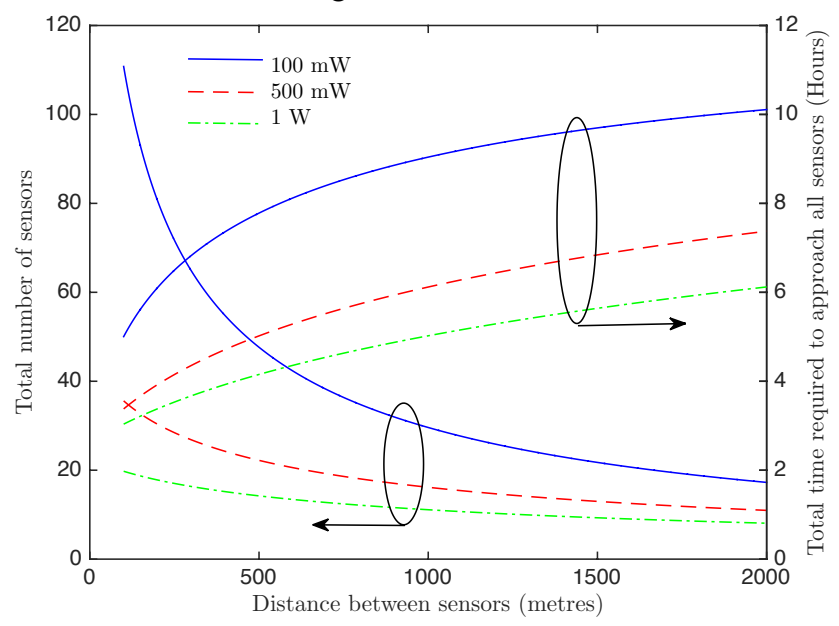

Figure 5 - Total number of sensors and total time required for the AUV to approach all sensors, as a function of the distance between sensors, for different average sensor power consumption values of $100 \mathrm{~mW}, 500 \mathrm{~mW}$ and $1 \mathrm{~W}$.

In order to provide further insight into how the AUV spends its energy during the whole operation period, we performed an energy breakout analysis, where we evaluated the energy spent per network segment as a function of the distance between sensors, for different average sensor power consumption values of $100 \mathrm{~mW}, 500 \mathrm{~mW}$ and $1 \mathrm{~W}$, and describing the different contributions between the energy spent travelling and the energy spent at the sensor node for hovering and wireless power transfer, as shown in Figure 6.

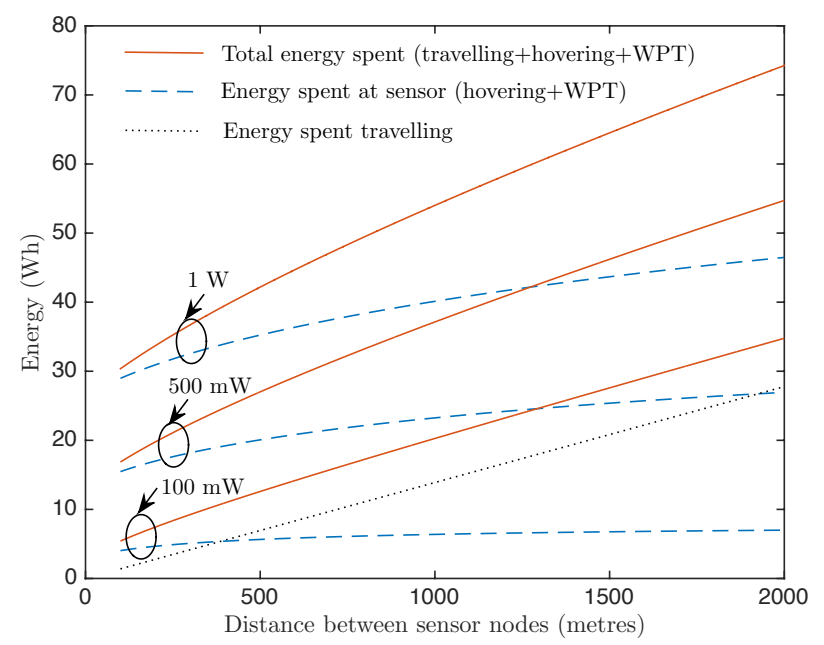

Figure 6 - Energy spent per sensor node as a function of the distance between sensors, for different average sensor power consumption values of $100 \mathrm{~mW}$, $500 \mathrm{~mW}$ and $1 \mathrm{~W}$, and describing the different contributions between the energy spent travelling and the energy spent at the sensor node for hovering and wireless power transfer.

For the case of $100 \mathrm{~mW}$ of sensor power consumption and a separation distance of 100 metres we can visit a total of approximately 110 sensors, while for a separation distance of 2000 metres, we would only be able to visit a total of approximately 18 sensors. In the same case, for a network of sensors with 1000 metres of distance between them, the total 
number of sensors that can be visited in one operation period is around 30, and the following energy breakout is achieved: 20 Wh of energy spent per segment $(20 \mathrm{Wh} \times 30$ sensors $=600 \mathrm{Wh}$ of AUV battery capacity), where $70 \%$ corresponds to the energy required to travel between sensors, $30 \%$ corresponds to the WPT energy plus hovering energy. As a preliminary conclusion, we can observe that for $100 \mathrm{~mW}$ of sensor power consumption, and above 400 metres of sensor separation, the limiting factor becomes the energy required to travel between sensors. However, this conclusion does not hold for the analyzed power consumptions of $500 \mathrm{~mW}$ and $1 \mathrm{~W}$, where the limiting factor is the energy spent at the sensors, both for hovering and WPT.

Finally, in order to better understand the different contributions of energy expenditure while the AUV is at the sensor, we performed an analysis where we analyzed the energy spent hovering only and compared it with the energy spent hovering plus WPT, as a function of the distance between sensors, for different average sensor power consumption values of $100 \mathrm{~mW}$, $500 \mathrm{~mW}$ and $1 \mathrm{~W}$, as shown in Figure 7.

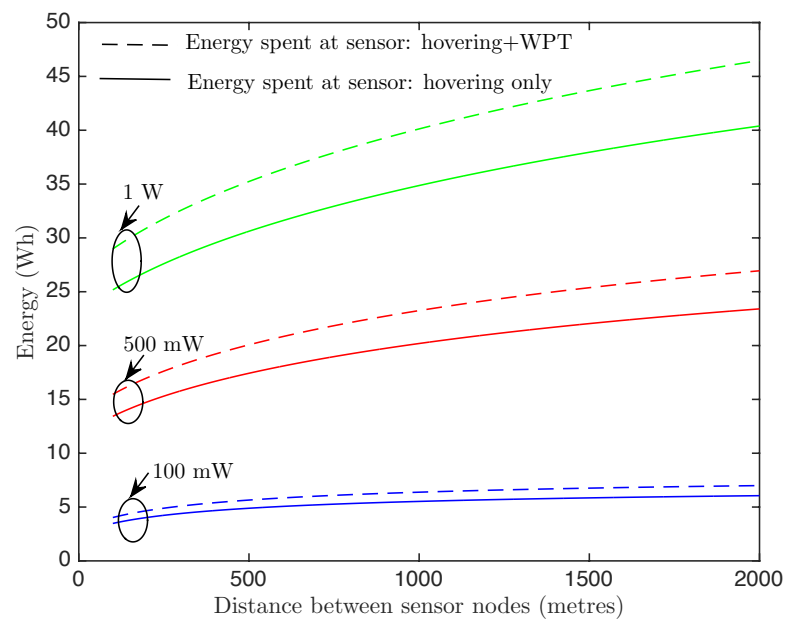

Figure 7 - Energy spent at sensor as a function of the distance between sensors, for different average sensor power consumption values of $100 \mathrm{~mW}, 500 \mathrm{~mW}$ and $1 \mathrm{~W}$, and describing the different contributions between the energy spent at the sensor. We represent in solid line the energy spent hovering only and in dashed line the energy spent hovering plus the energy spent for WPT.

As it can be observed, the major contribution of the energy spent at the sensor results from the energy required for AUV hovering, due to the increased time the AUV needs to be near the sensor providing energy. The difference between the two results presented corresponds essentially to the energy required for WPT (since the energy required for OWC based data transfer is almost insignificant due to the short duration). This difference increases for higher sensor power consumption as expected. Therefore, one can conclude that improving (reducing) the AUV hovering power, or devising ways of being able to turn off the AUV motors would be important, especially in scenarios where the average sensor power consumption is higher than $100 \mathrm{~mW}$. For average sensor power consumption values of $100 \mathrm{~mW}$ or less, and provided the distance between sensors is higher than 400 metres, it would be important to work on techniques to improve (reduce) the AUV power consumption at cruise speed.

\section{CONCLUSIONS}

In this paper we evaluated the long-term deployment feasibility of a large-scale network of abandoned underwater sensors, with power provided by AUVs in periodic visits. It was concluded that improving (reducing) the AUV hovering power, or devising ways of being able to turn off the AUV motors would be important, especially in scenarios where the average sensor power consumption is higher than $100 \mathrm{~mW}$. For average sensor power consumption values of $100 \mathrm{~mW}$ or less, and for distances between sensors higher than 400 metres, it would be important to work on techniques to improve (reduce) the AUV power consumption at cruise speed.

\section{ACKNOWLEDGMENT}

This work is financed by the ERDF - European Regional Development Fund through the Operational Programme for Competitiveness and Internationalisation - COMPETE 2020 Programme and by National Funds through the Portuguese funding agency, FCT - Fundação para a Ciência e a Tecnologia within project POCI-01-0145-FEDER-031971.

\section{REFERENCES}

[1] T. Ressurreição, F. Gonçalves, C. Duarte, R. Gonçalves, R. Gomes, R. Santos, R. Esteves, P. Pinto, I. Oliveira, L. M. Pessoa, System Design for Wireless Powering of AUVs, in Proc. of OCEANS 2017, Jun. 2017.

[2] M. Khalighi, C. Gabriel, B. Silva, L.M. Pessoa, Underwater visible light communications, channel modelling and system design, Chapter in Visible Light Communications: Theory and Applications, Jun. 2017, ISBN: 9781498767538.

[3] N. A. Cruz and A. C. Matos, "The MARES AUV, a Modular Autonomous Robot for Environment Sampling," in OCEANS 2008, Sept 2008, pp. 16.

[4] Figueiredo, Andre B., Bruno M. Ferreira, and Aníbal C. Matos. "Visionbased localization and positioning of an AUV." In OCEANS 2016Shanghai, pp. 1-7. IEEE, 2016. 